\title{
TRANSIENT AERO-THERMO-MECHANICAL MULTIDIMENSIONAL ANALYSIS OF A HIGH PRESSURE TURBINE ASSEMBLY THROUGH A SQUARE CYCLE
}

\author{
Vlad Ganine; John W. Chew, Nicholas J. Hills \\ Thermofluids Systems UTC \\ Faculty of Engineering and Physical Sciences \\ University of Surrey \\ Guildford, UK
}

\author{
Sulfi N. Mohamed, \\ Rolls-Royce plc \\ Derby, UK
}

\author{
Matthew M. Miller, \\ Rolls-Royce Corporation \\ Indianapolis, IN, USA
}

\begin{abstract}
Better understanding and more accurate prediction of heat transfer and cooling flows in aero engine components in steady and transient operating regimes are essential to modern engine designs aiming at reduced cooling air consumption and improved engine efficiencies. This paper presents a simplified coupled transient analysis methodology that allows assessment of the aerothermal and thermomechanical responses of engine components together with cooling air mass flow, pressure and temperature distributions in an automatic fully integrated way. This is achieved by assembling a fluid network with contribution of components of different geometrical dimensions coupled to each other through dimensionally heterogeneous interfaces. More accurate local flow conditions, heat transfer and structural displacement are resolved on a smaller area of interest with multidimensional surface coupled CFD/FE codes. Contributions of the whole engine air-system are predicted with a faster mono dimensional flow network code. Matching conditions at the common interfaces are enforced at each time step exactly by employing an efficient iterative scheme. The coupled simulation is performed on an industrial high pressure turbine disk component run through a square cycle. Predictions are compared against the available experimental data. The paper proves the reliability and performance of the multidimensional coupling technique in a realistic industrial setting. The results underline the importance of including more physical details into transient thermal modelling of turbine engine components.
\end{abstract}

*Address all correspondence to v.ganine@ surrey.ac.uk.

\section{NOMENCLATURE}

$A$ Cross-sectional area

$A_{s}$ Surface area

B Matrix of cross-sectional area variation effects

C Matrix of friction effects

$\mathscr{C}$ Heat capacity

$C_{d}$ Aerodynamic drag force coefficient

$C_{e f f}$ Effective seal clearance

$C_{n}$ n-th fin radial clearance

$\mathscr{C}_{S}$ Solid heat capacity

$\mathscr{C}_{p}$ Specific heat capacity at constant pressure

$C_{w}$ Non-dimensional mass flow rate

$\mathscr{F}$ Total flux

$\mathbb{F}$ Fluid solver

$\mathscr{K}$ Thermal conductivity coefficient

$M$ Mach number

Q Heat flux vector

$R e_{x}$ Axial Reynolds number

$R e_{\theta}$ Rotational Reynolds number

$\mathbb{S}$ Solid structural solver

T Temperature vector

$\mathbb{T}$ Solid thermal solver

$\mathbf{U}$ Vector of conservative variables

$U_{b}$ Bulk axial velocity

$c$ Speed of sound

d Displacement vector

$h$ Heat transfer coefficient

$d_{h}$ Hydraulic diameter at inlet

$d_{r}$ Radial displacement 
$l$ Length

n Normal exterior

p Pressure vector

$\tilde{p}$ Pressure on fictitious boundary

$r_{i}$ Radius at the cone entry

$r$ Local radius

$t$ Time

u Velocity vector

- Inner product

$\nabla$ Gradient of a vector field

$\partial$ Differentiation

\section{Greek}

$\Omega$ Domain

$\boldsymbol{\varepsilon}$ Elastic strain tensor

$\Gamma^{c}$ Averaged quantity boundary

$\Gamma^{w}$ Wall boundary

$\varepsilon$ Residual tolerance

$\boldsymbol{\varepsilon}$ Elastic strain tensor

$\mu$ Dynamic viscosity

$\dot{\mu}$ Mass flow rate vector

$\rho$ Density

$\pi$ Pi number

$\boldsymbol{\sigma}$ Elastic stress tensor

$\tau$ Viscous stress tensor

$\omega$ Rotational speed

\section{Superscripts}

() down Downstream

()$^{n}$ n-th inner iteration

()$^{m}$ m-th outer iteration

() ${ }^{\text {rot }}$ Rotor

() ${ }^{\text {stat }}$ Stator

()$^{T}$ Transpose

() $)^{\text {up }}$ Upstream

\section{Subscripts}

() $1 D$ Defined on $1 D$ domain

() $c$ Computed

()$_{f}$ Defined on fluid domain

()$_{i}$ Defined on inflow boundary

()$_{k}$ k-th time step

() $m$ Measured

()o Defined on outflow boundary

() $s$ Defined on solid domain

()$_{w}$ Defined on wall interface

()$_{\Gamma}$ Defined on interface $\Gamma$

()$_{\theta}$ Tangential component

()$_{0}$ Undeformed configuration

||| Norm

\section{Acronyms}

CFD Computational fluid dynamics

HPT High Pressure Turbine

HPC High Pressure Compressor

$N G V$ Nozzle guide vane

$N H$ High pressure angular velocity

$N I$ Intermediate pressure angular velocity

$N L$ Low pressure angular velocity

\section{INTRODUCTION}

Design of modern lighter, quieter and more energy-efficient aero engines constantly evolves towards higher achievable cycle temperatures and pressures. This path of evolution aiming at improved cycle thermal efficiencies, however, is limited by the material temperature of the hot gas path components. Development of newer and more effective cooling systems is an increasingly challenging task because the amount of air diverted for cooling must be kept at lowest possible levels. An important challenge here is accurate knowledge of thermal transient behavior of gas turbine components, which is known less accurately compared to the steady-state response. If more precise transient predictions were available, expensive engine testing could be avoided because the detrimental effects of heat soakage or clearance changes could be known earlier at design stage.

Preliminary design procedures traditionally combine fast flow network tools based on simplified quasi- $1 D$ methods [1] with FE heat conduction codes equipped with empirical heat transfer correlations. Physics-based simulation methods employ $2 \mathrm{D} / 3 \mathrm{D}$ CFD codes to provide more accurate and reliable description of the aero-thermal state of turbine engine components. Owing to high computational costs earlier efforts were mostly limited to steady analyses at the component level $[2,3,4,5]$. Transient conjugate heat transfer analysis to capture heat flux and wall temperature distribution were carried out in $[6,7,8,9,10,11]$. In the majority of cases the literature reports a reasonable level of agreement with experimental data at both steady-state and transient operating conditions.

A large number of problems often involve several coupled phenomena such as flow, heat transport and mechanical deformations. To improve predictive accuracy at transient operating conditions the effects of structural deformation on aero-thermal state must be accounted for. Fully coupled CFD-FE aero-thermomechanical multiphysics simulations with automatic fluid mesh adaptation under structural deformations have been performed in $[12,13]$. The results showed physically consistent variations in thermal response at both steady-state and transient operating conditions originated in engine solid domain deflections, but the accuracy largely relied on the fidelity of the upstream and downstream boundary conditions. Moreover, higher computational cost of resolving flow features and temperatures between thermally coupled fluid and solid domains as well as the difficulties of meshing complex 3D surfaces of labyrinth seals (ex. abrad- 
able honeycomb landings) restrict practical application of this approach. To reduce the cost of transient aero-thermo-mechanical simulation many authors resort to coupling the quasi- $1 D$ fluid and FEA thermal and stress analysis codes $[14,15]$.

Full transient resolution of a whole engine model using higher fidelity coupled 2D/3D CFD and FEA solvers is currently not practical due to extreme computational costs, which largely prevents their integration into broader system level and multidisciplinary studies. The cost of solving expensive high-fidelity fluid models can be lowered by using a multi-domain dimensionally heterogenous coupled approach. More accurate temporal or spatial behavior is resolved on multiple smaller areas of interest with $2 D / 3 D$ fluid models, and the contribution of peripheral areas or areas with a single dominant space dimension can be represented by lower fidelity $1 D$ models. In this way the feedback mechanisms, the reciprocal influence of local dynamics on global system behavior essential for the accuracy of numerical simulations and ultimately for their impact on design are retained in the coupled model.

The major challenge of such an approach is numerical coupling between many dimensionally heterogeneous models with incompatible interfaces in such a way that it ensures the stability of the solution process and guarantees that the transferred quantities are conserved on all coupled interfaces. Simple underrelaxation techniques are not applicable in this context due to convergence difficulties. The latter stem from strong hydraulic coupling between the multiple dimensionally heterogeneous components assembled into a single flow network and from strong thermal coupling that exists between the fluid and the solid surfaces.

This paper presents the development and application of a dimensionally heterogeneous multiscale coupling technique in the context of transient aero-thermo-mechanical analysis of gas turbine engine components. We build upon the aero-thermal methods developed in $[7,8]$, the more recent work on coupling of $1 D$ fluid and $2 D$ solid models in [16] and the simplified modeling of protruding $3 D$ features in [17]. A detailed validation has been carried out on an industrial high pressure turbine model simulated through a full transient cycle coupled to an air system model of a whole engine. The proposed coupling technique is shown to converge robustly at all operating conditions. The accuracy of simulated results is compared to measured data. Computational cost of the proposed coupling approach is discussed and compared to the cost of a simpler aero-thermal CFD-FEA analysis.

\section{METHODOLOGY}

A brief description of the general formulation for weak coupling between the dimensionally heterogeneous fluid flow models and the solid models describing structural deflection and heat transfer as well as iterative solution technique are given in the following.

\section{Multiphysics Coupling Formulation}

The global domain is subdivided into the non-overlapping fluid $\Omega_{f}$ and solid $\Omega_{s}$ subdomains sharing a common interface. The fluid subdomain is further subdivided into the partitions described by the higher $\Omega_{f_{2 D}}$ and lower $\Omega_{f_{1 D}}$ dimensional models (without loss of generality we assume $2 D$ and $1 D$ ).

Flow in the high dimensional fluid subdomain is governed by the compressible Reynolds-averaged Navier-Stokes equations written in a short form as:

$$
\frac{\partial \mathbf{U}}{\partial t}+\nabla \cdot \mathscr{F}(\mathbf{U}, \nabla \mathbf{U})=0 \text { in } \Omega_{f_{2 D}}
$$

Evolution of the solid subdomain deflection is governed by the elastodynamic equation:

$$
\rho \frac{\partial^{2} \mathbf{d}_{s}}{\partial t}-\nabla \cdot \boldsymbol{\sigma}_{s}\left(\boldsymbol{\varepsilon}_{s}, T_{s}\right)=0 \text { in } \Omega_{s}
$$

Heat transfer in the solid domain is described by the thermal equilibrium equation assuming the Fourier's law:

$$
\rho \mathscr{C}_{s} \frac{\partial T_{s}}{\partial t}-\nabla \cdot\left(\mathscr{K} \nabla T_{s}\right)=0 \text { in } \Omega_{s}
$$

The fluid model of reduced dimension retains only a single coordinate associated with the dominant flow direction. The conservative form of such quasi-one-dimensional continuity, momentum and energy equations is:

$$
\frac{\partial \mathbf{U}(l, t)}{\partial t}+\frac{\partial \mathscr{F}(\mathbf{U})}{\partial l}+\mathbf{B}(\mathbf{U})+\mathbf{C}(\mathbf{U})=0 \text { in } \Omega_{f_{1 D}}
$$

Since the spatial resolution of the above 1D fluid model is typically too coarse for detailed description of the temperature field on solid boundaries an auxiliary $1 D$ energy equation is normally solved on the solid subdomain boundary. It is typically implemented in a form of the thermal boundary condition of FE solver:

$$
\rho \mathscr{C}_{p} \frac{\partial T}{\partial t} A+\rho \mathscr{C}_{p} u \frac{\partial T}{\partial l} A=h \frac{\partial A_{s}}{\partial l}\left(T_{s}-T\right) \text { in } \Omega_{f_{1 D}}
$$

To combine the dimensionally heterogeneous problems into a single system we need to enforce appropriate matching conditions employing some simplifying assumptions due to geometrical mismatch. One of the most common choices [18] is to impose the continuity of the mean pressure and flow rate on the coupling interfaces. We need to assume that the pressure on the interface is constant and the boundary is perpendicular to the main flow direction, so that the normal viscous stress can be discarded [19]. Since $1 D$ model computes only average-in-space axial velocity we assume a spatially uniform velocity profile on the interface with a total flow corresponding to one of the $1 D$ model. Similarly, for thermal coupling we assume a spatially uniform temperature 
profile on $\Gamma^{c}$.

$$
\begin{array}{lll}
p_{1 D}=\frac{1}{|\Gamma|} \int_{\Gamma} p_{2 D} d \gamma & \text { on } \quad \Gamma^{c} \\
\dot{\mu}_{1 D}=-\rho \int_{\Gamma} \mathbf{u}_{2 D} \cdot \mathbf{n} d \gamma & \text { on } \quad \Gamma^{c} \\
T_{1 D}=\frac{1}{|\Gamma|} \int_{\Gamma} T_{2 D} d \gamma & \text { on } \quad \Gamma^{c}
\end{array}
$$

The continuity of temperatures, velocities, and the normal components of heat flux and traction coupling conditions are satisfied on the common interface between the solid and the fluid subdomains $\Gamma^{w}$. In this work we assume that solid domain velocity and acceleration terms are small enough to be discarded (the quasistatic assumption). With these approximations the interface conditions become:

$$
\begin{aligned}
\mathbf{n}_{s} \cdot \boldsymbol{\sigma}_{s} & =-\mathbf{n}_{f} \cdot(p \boldsymbol{I}+\boldsymbol{\tau}) & & \text { on } \Gamma^{w} \\
\mathbf{u}_{s} & =\mathbf{0} & & \text { on } \Gamma^{w} \\
\mathbf{T}_{s} & =\mathbf{T}_{f} & & \text { on } \Gamma^{w} \\
\mathbf{n}_{s} \cdot \mathbf{q}_{s} & =-\mathbf{n}_{f} \cdot \mathbf{q}_{f} & & \text { on } \Gamma^{w}
\end{aligned}
$$

Note that a non-zero tangential velocity component of the rotating solid wall boundary can be prescribed at the fluid domain boundary. The geometric compatibility (kinematic) conditions are enforced only on $\Gamma_{1 D}^{w}$ boundaries where the relative displacement of the solid $\Omega_{s}^{r o t}$ and $\Omega_{s}^{\text {stat }}$ subdomains are expected to affect the fluid solution. By postulating a cylindrical shape of the fluid interface the cross-sectional area compatibility can be expressed as:

$$
\pi\left(r_{0}^{r o t}+d_{r}^{r o t}\right)^{2}-\pi\left(r_{0}^{s t a t}+d_{r}^{s t a t}\right)^{2}=A_{1 D} \text { on } \Gamma^{w}
$$

where $r_{0}^{r o t}$ and $r_{0}^{\text {stat }}$ represent the undeformed configuration. The simplifying assumptions of the $2 D$ model boundary conditions on $\Gamma^{c}$ boundaries have a major impact on the spacial location of the inflow and outflow artificial boundaries. These need to be positioned at the points where flow can reasonably be approximated as $1 D$. This does not correspond to the modeling assumptions of the flow network model, which regularly calculates the averaged pressure inside the cavity, not at its outflow extremities. For that reason $2 D$ computational subdomains are artificially elongated into the $1 D$ subdomain forming an overlap between the dimensionally heterogeneous models. Fig. 1 shows a representative example of coupling between two solid $2 D$ axisymmetric subdomains $\Omega_{s}^{r o t}$ and $\Omega_{s}^{\text {stat }}$, two fluid $2 D$ axisymmetric subdomains $\Omega_{f}^{u p}$ and $\Omega_{f}^{\text {down }}$, and a $1 D$ fluid subdomain $\Omega_{f_{1 D}}$. The mean pressures to match are integrated on the inner artificial interfaces $\tilde{\Gamma}_{i n}^{c}$ and $\tilde{\Gamma}_{\text {out }}^{c}$, but the boundary conditions of the fluid $2 D$ problem need to be set on the outer $\Gamma_{\text {in }}^{c}$ and $\Gamma_{\text {out }}^{c}$ artificial boundaries. This creates an unknown uniform pressure boundary condition on $\Gamma_{\text {in }}^{c}$ or $\Gamma_{\text {out }}^{c}$ that needs to be evaluated implicitly at some additional computational cost. The dimensionally heterogeneous fluid problems are

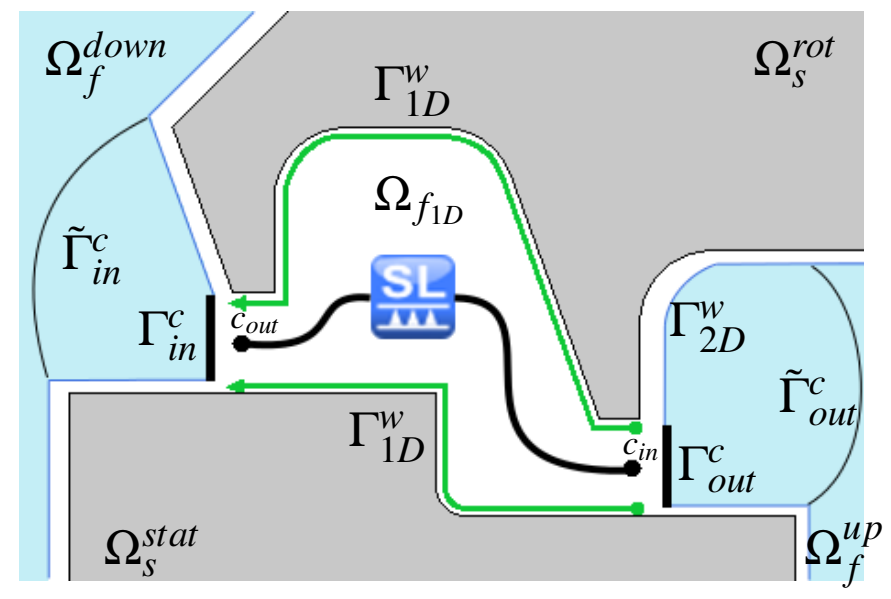

FIGURE 1. An example of 1D-2D multiscale coupling connection.

coupled at the interfaces corresponding to a cross section for the $2 D$ case $\Gamma_{\text {out }}^{c}$ and a single point for the $1 D$ model $c_{\text {in }}$. Point $c_{\text {out }}$ of the $1 \mathrm{D}$ model is in turn coupled to the downstream boundary $\Gamma_{\text {in }}^{c}$ of the $2 D$ problem.

\section{Iterative technique}

The $2 \mathrm{D}$ aerodynamics problem is discretized in space by the finite volume method. The solid elastic and thermal subproblems are discretized in space by FE method. The 1D flow problem is discretized into a number of control volumes represented by nodes interconnected by links. Mass and energy conservation equations are solved on the nodes and the momentum balance equation is solved on the links. The extra energy balance equation defined on the solid boundary in contact with one dimensional flow problem region is discretized using the integration points resulting from the FE discretization. Instead of time integration the coupled system of ODEs, we employ the quasi-steady fluid and structure assumptions [7, 8,20]. It is a frequently employed time saving mathematical modeling technique for processes with different time scales. On the remaining system of solid thermal ODEs we perform time discretization with the standard generalized trapezoidal rule.

The driving motivation of this work is to assemble a fluid network with the contribution of components of different geometrical dimension coupled through heterogeneous interfaces. Therefore we consider a further partition of $\Omega_{f}$ into several higher dimensional $\Omega_{f_{2 D}^{1}}, \Omega_{f_{2 D}^{2}}, \ldots, \Omega_{f_{2 D}^{n}}$ and lower dimensional $\Omega_{f_{1 D}^{1}}, \Omega_{f_{1 D}^{2}}, \ldots, \Omega_{f_{1 D}^{m}}$ subdomains. It is practical to assume that no higher dimensional components are directly in contact with 
each other. The heterogeneous interfaces are given by a set of the inflow boundaries $\Gamma_{i n, i}^{c}, i=1, \ldots, N_{i n}$, the outflow boundaries $\Gamma_{\text {out }, j}^{c}, j=1, \ldots, N_{\text {out }}$ and a set of their respective matching points $c_{\text {out }, i}, i=1, \ldots, N_{\text {in }}$ and $c_{i n, j}, j=1, \ldots, N_{\text {out }}$. We distinguish between the inflow and the outflow boundaries in a sense that the mean flux $\mathbf{u} \cdot \mathbf{n}$ averaged in space is expected to be negative at an inflow and positive at an outflow boundary.

To obtain a stable and computationally efficient algorithm we employ a classical Dirichlet-Neumann partitioning scheme on the fluid-solid interfaces. The fluid subproblems is supplemented by the Dirichlet boundary condition (temperature), and the Neumann condition (heat flux) is imposed on the solid subproblem [21,22]. On the coupled boundaries between the dimensionally heterogeneous fluid models we prescribe the Dirichlet boundary conditions (velocities) at the inflow interfaces of the 2D models, and the Neumann boundary conditions (pressures) at the outflow interfaces. Temperatures are prescribed at the inflow boundaries of the 2D subdomains, the normal component of heat flux is assumed to be zero at the outflow boundaries. Using a compact notation for the time and space discrete stand-alone subproblems we can write the coupled nonlinear interface problem as:

$$
\begin{cases}\left(\boldsymbol{Q}_{w}, \boldsymbol{p}_{w}, \dot{\boldsymbol{\mu}}_{o}, \boldsymbol{T}_{o}, \boldsymbol{p}_{i}\right) & =\mathbb{F}_{2 D}\left(\boldsymbol{T}_{w}, \dot{\boldsymbol{\mu}}_{i}, \boldsymbol{T}_{i}, \boldsymbol{p}_{o}, \boldsymbol{\Delta} \boldsymbol{p}_{o}\right) \\ \left(\dot{\boldsymbol{\mu}}_{i}, \boldsymbol{T}_{i}, \boldsymbol{p}_{o}\right) & =\mathbb{F}_{1 D}\left(\dot{\boldsymbol{\mu}}_{o}, \boldsymbol{T}_{o}, \boldsymbol{p}_{i}, \boldsymbol{A}\right) \\ \left(\boldsymbol{T}_{w}\right) & =\mathbb{T}_{2 D}\left(\boldsymbol{Q}_{w}\right) \\ (\boldsymbol{A}) & =\mathbb{S}_{2 D}\left(\boldsymbol{T}_{w}, \boldsymbol{p}_{w}\right)\end{cases}
$$

Note that vectors $\boldsymbol{p}_{i}$ and $\boldsymbol{p}_{o}$ group together pressures averaged on the inner artificial inflow and outflow boundaries $\tilde{\Gamma}^{c}$, whereas vector $\Delta \boldsymbol{p}_{o}$ combines differentials between the pressures averaged on the inner artificial $\tilde{\Gamma}_{\text {out }}^{c}$ and the outer real $\Gamma_{\text {out }}^{c}$ outflow boundaries correspondingly. To remove this extra unknown from the nonlinear system we can reasonably approximate it by taking from the previous time step.

The system is solved in an iterative loop by a Gauss-Seidel like scheme in a nested iterative implicit algorithm illustrated schematically in Fig. 2. Three loops are iterated over in order to solve a fully implicit coupled aero-thermo-mechanical problem. The first loop defines the global time stepping sequence, the second loop is needed to perform iterations between the weaker coupled structural dynamics problem and the remaining part of the system. At the innermost loop level we resolve stronger coupled thermal fluid-structure interactions and coupled dimensionally heterogeneous fluid subproblems. The coupling strength is viewed here in the numerical sense as the degree of subsystem interaction. The coupling is viewed strong if the solution of one field strongly depends on the accuracy of the coupling terms calculation [23]. Every inner iteration $n$ all the $1 D, 2 D$ fluid subproblems and $2 D$ solid thermal subproblem are solved to full convergence in a sequence. The inner loop iterates on the discrete solution vector $\left[\boldsymbol{T}_{w}^{(n)}, \dot{\boldsymbol{\mu}}_{i}^{(n)}, \boldsymbol{T}_{i}^{(n)}, \boldsymbol{p}_{o}^{(n)}\right]^{T}$ until the iteration residual falls below the prescribed tolerance. The solid domain displace- ments used to evaluate the cross sectional area $\boldsymbol{A}^{(m)}$ are kept fixed. Once all the fields are balanced at the inner loop level we invoke the structural elastic solver and $1 D$ flow solver every outer iteration $m$. In general, at least two outer iterations are needed. At the outer iteration level the solution vector is augmented with the cross section area. This guarantees that the full four field problem is balanced if and only if all the prescribed tolerances are satisfied.

Due to the heterogeneous nature of this problem with strongly interacting physical fields, the methods to stabilize and accelerate iterations are required. A simple under-relaxation iterative procedure applied to this problem does not guarantee convergence. More robust strong coupling techniques are based on reinterpreting the original coupling problem as an interface problem. This allows to apply Jacobian-free Broyden-type or NewtonGMRES methods. To accelerate and stabilize the inner iteration loop here we employ the Anderson acceleration technique [24]. It was previously shown to be particularly effective on thermally coupled problems [25]. One option is to group the wall temperatures together with the averaged quantities such as mass flows, pressures and fluid temperatures into a single nonlinear interface problem and solve it using the Anderson method. But in absence the strong mutual coupling of the wall temperatures $\boldsymbol{T}_{w}$ and the averaged flow variables $\dot{\boldsymbol{\mu}}_{i}, \boldsymbol{T}_{i}, \boldsymbol{p}_{o}$ the inverse Jacobian entries estimated at each iteration may be susceptible to the numerical noise in thermal predictions. Therefore simultaneous solution of two smaller uncoupled fixed-point interface problems each accelerated by the Anderson technique leads to a faster converging method:

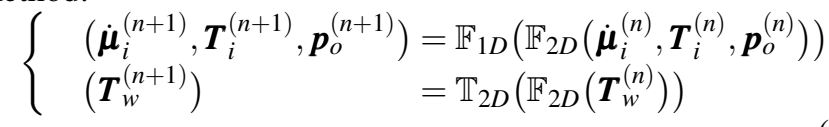

\section{TEST CASE DESCRIPTION}

Fig. 3 shows the radial cross section of the HP turbine disk of a modern three spool aeroengine used in the present study. Compressed air from the last stage of the HP compressor enters the drive cone cavity radially through the main annulus inlet. It exits the cavity through a succession of labyrinth seals supplying air to the pre-swirl cavities. In addition, cooling air enters the preswirl vanes and nozzles from the combustor burner bypass. From there it proceeds to HP turbine blades for internal and film cooling. Some of the air from the pre-swirl chamber exits to maintain a positive purge flow into the main annulus preventing hot gas ingestion into the front rim cavity. The turbine rear cavity is supplied with pre-swirled air from the HP-IP box structure to provide sealing flow to the rear rim seal cavity as well as cooling flow to the turbine disk. The bore, the front and the rear inner cavities supply cooling flow to the inner part of the HPT disk.

The solid subdomain is modeled with a $2 D$ axisymmetric representation of the $3 D$ geometry. It is discretized into 15,143 


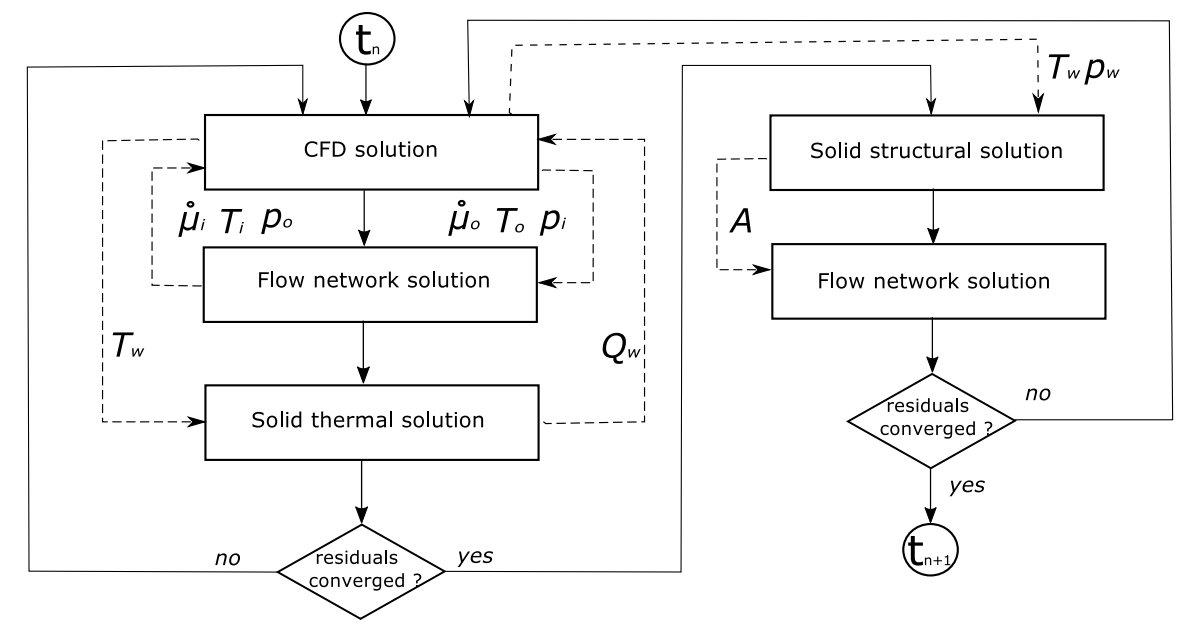

FIGURE 2. Flow chart of the nested iterative scheme.

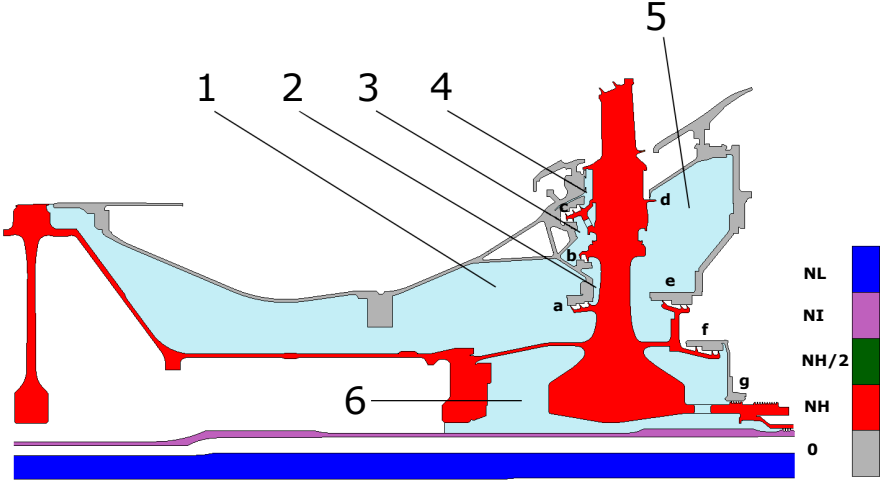

FIGURE 3. Geometrical layout of the high pressure turbine FE solid and CFD fluid models. The CFD domain includes: 1 - the drive cone cavity, 2 - the pre-swirl inner cavity, 3 - the pre-swirl chamber cavity, 4 - the front rim cavity, 5 - the rear cavity, 6 - the inner front, the bore and the inner rear cavities. The FE domain includes the following seals: (a) inner-inner seal, (b) - inner-outer seal, (c) - outer-inner and outer-outer seals, (d) - knife seal, (e) - HP triple seal (f) - HP-IP seal, (g) - bearing chamber seals.

triangular elements. The proprietary FEA code SC03 [26] employed in this work reuses the same computational grid for both stress and transient heat transfer analyses. The code comes with all specialists convective and radiative thermal boundary conditions. To reduce the analysis time the original FE model of the whole engine is partitioned into subcomponents. At the HPT subcomponent's extremities we impose the axial and radial displacements read across from the whole engine FE model predictions. The solid model calculates axial and radial displacements of 12 labyrinth and knife seals. These are used to calculate the equivalent areas available to flow consequently employed in the coupled simulation.

On the uncoupled to CFD boundaries we place thermal convective boundary conditions with the standard heat transfer correlations. They have been obtained following the back-to-back comparison to measured thermocouple data. The model matching procedure is described in [27]. The convective boundary conditions are set up by specifying their flow direction, the starting air temperature, the windage power generation and the heat transfer coefficient. The starting air temperatures are supplied from the engine performance data. They can also be linked to exit air temperatures of the upstream CFD models or other thermal boundary conditions.

The transient engine cycle is specified in the $\mathrm{SC} 03$ code by defining the time histories of main annulus conditions such as temperatures, pressures and mass flow rates together with the structural loads. The square cycle used in our simulation is shown in Fig. 4 in terms of the high pressure turbine normalized angular velocity. The engine is run through a succession of stabilized low

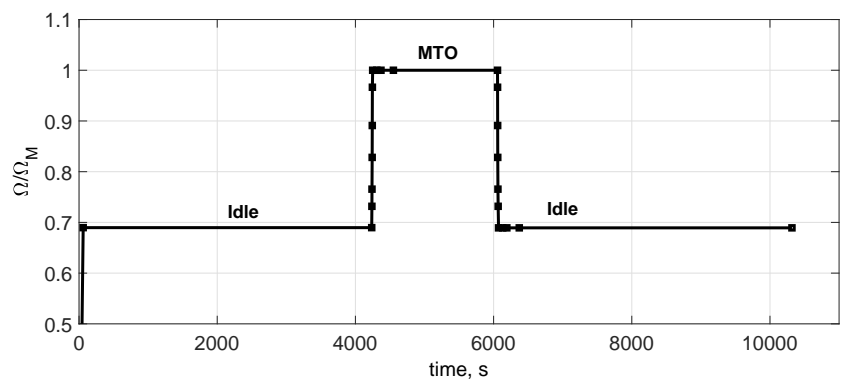

FIGURE 4. Flight cycle definition in terms of high pressure turbine angular velocity. 
power (idle) condition, a rapid acceleration to high power (maximum take-off) condition, stabilized high power condition, and a rapid deceleration back to the low power condition.

For $1 D$ fluid analysis we use a proprietary industrial fluid network solver SPAN. The flow network model of the whole engine secondary flow system is discretized into 461 nodes interconnected by 685 links. The flow and the heat transfer links contain 1850 bits. The nodes represent large volumes of air and the bits define pressure or heat losses within a link. The model contains 83 boundary condition nodes supplied with time varying temperatures and pressures from the engine main annulus (performance data). There are many types of bits available to accurately describe the flow behavior in a turbine engine internal air system. They could account for the unmodelled multidimensional turbulent flow effects in cavities or for pumping and friction losses due to rotation. Many empirical loss factors can be found in the standard references: static or rotating orifice bits, seal bits, vortex bits, restriction or pipe friction loss bits. Non-standard effects are modeled with flow characteristic bits using experimental correlations. Some of the bits depend on parameters that are kept constant during simulation. Others are parameterized dynamically by changing their geometrical properties as the simulation progresses, such as areas or radial seal clearances for example.

In contrast to the simpler one-dimensional flows the velocity has to be treated as a two dimensional vector with the streamwise and the circumferential components. The streamwise velocity is computed from the balance equation. The circumferential velocity is modeled using empirical or theoretical correlations.

The internal cavities selected for $2 D$ CFD analysis are modeled as $2 D$ axisymmetric. The use of the axisymmetric assumption involves some geometric approximations. $3 D$ features such as nozzles and holes are approximated with annular slots of the same effective areas, the effects of $3 D$ bolts and nuts are approximated with the body force model discussed in the foregoing. The fluid computational domain has been subdivided into 6 separate models. The extent of CFD domains and the locations of inflow and outflow boundaries are presented in Fig. 5, which also shows the flow fields at low and high power stabilized conditions.

No-slip boundary conditions are applied to all walls. At the inflow boundary of the drive cone cavity we specify the total pressure, at other cavities' inlets we prescribe the mass flow rate. The total temperature, the incoming flow angle and the tangential velocity component are prescribed at all inflow boundaries, while the static pressure is enforced at all outflow boundaries. All conditions but the one at the upstream inlet boundary of the drive cone cavity are computed at run time and updated through coupling with $1 D$ models. Only conditions at the inlet of the drive cone cavity evolve according to the main annulus performance data. Relative locations of the natural inflow and outflow boundaries and the artificial boundaries created to exchange pressure transfer conditions with 1D models are depicted in Fig. 6.

The fluid subdomains are meshed with the multi-block struc- tured quadrilateral cells using ICEM CFD. Two sets of grids were created in order to maintain proper boundary layer resolution at two distinct operating regimes. For all grids, the first cell of the wall was held to a non-dimensional near wall spacing $y+$ between 20 and 200 for use with the with the standard $\kappa-\varepsilon$ turbulence model and wall functions. For brevity, mesh sensitivity studies are not presented here. The grid independence was assessed by comparing swirl velocity profiles, surface heat flux distributions and the averaged quantities at the outflow boundaries. The grid resolution and the choice of turbulence model are consistent with our previous experience $[28,8,29]$. Grid sizes for all six cavities at both operating regimes are given in Tab. 1. Steady flow field adiabatic solutions were obtained and examined on both sets of grids to ensure convergence. For all cavities and flow regimes the rootmean-squares of normalized residuals were less than $1 \times 10^{-12}$. The mass imbalances were less than $0.03 \%$ of the inflow. The angular momentum imbalances were less than $2 \%$ of the walls and rotating features torque. Energy imbalances were less than $2 \%$ of the difference in total enthalpy between inlets and outlets.

During the engine cycle simulation fluid flow is computed switching between two sets of grids mid-way between the idle and the maximum take-off speeds.

The aerodynamic flow conditions for the two engine conditions are described in Tab. 2 in terms of the rotational Reynolds number $R e_{\theta}=\rho r_{i}^{2} \omega / \mu$ based on the inlet radius $r_{i}$, the axial Reynolds number $\operatorname{Re}_{x}=\rho U_{b} d_{h} / \mu$, the Mach number $M=U_{b} / c$, and the turbulent flow parameter $\lambda_{T}=C_{w} R e_{\theta}^{-0.8}$, where nondimensional mass flow rate $C_{w}=\dot{\mu} / \mu r_{i}$.

The computation is executed using an industrial finite volume edge-based compressible RANS code Hydra. The code works on unstructured hybrid grids, data are stored at a cell vertex and dual control volumes are defined around each vertex using a vertexcentered approach. This allows for a second order accurate spatial discretization using Roe's scheme. An explicit Runge-Kutta scheme is used to advance the solution in time, dual time stepping with preconditioning and multigrid employed for convergence acceleration to steady state. Further details may be found in [30]. The coupling between the three codes with the interface solver have been implemented as a dynamic library SC89 within the FEA code $\mathrm{SC} 03$.

Body force modeling The non-rotating nonaxisymmetrical features in the drive cone cavity are modeled with the simplified body force model described in [17]. The main idea is to replace the $3 D$ features by an axisymmetric body force field that brings commensurate changes in angular momentum to the flow. The drag associated with the $3 D$ nuts and bolts is modeled by a source term in the momentum equations. The inertial resistance of the protruding $3 D$ features in circumferential direction of the cylindrical coordinate system can be expressed 


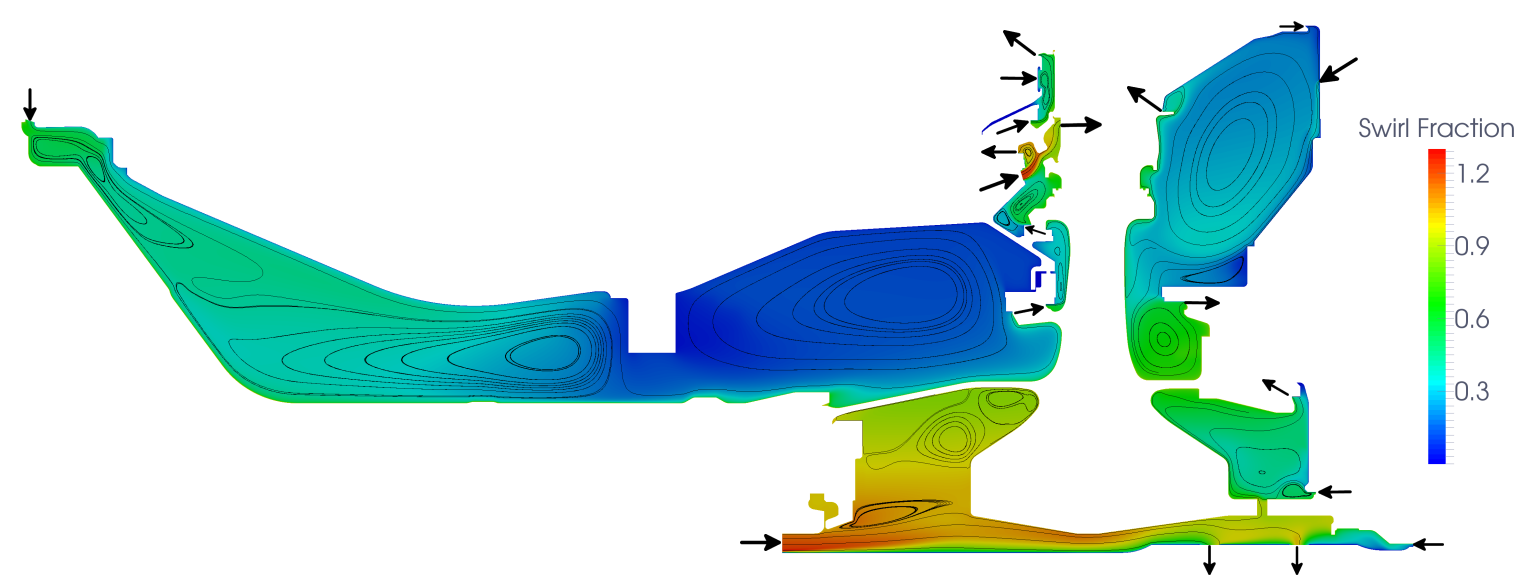

(a)

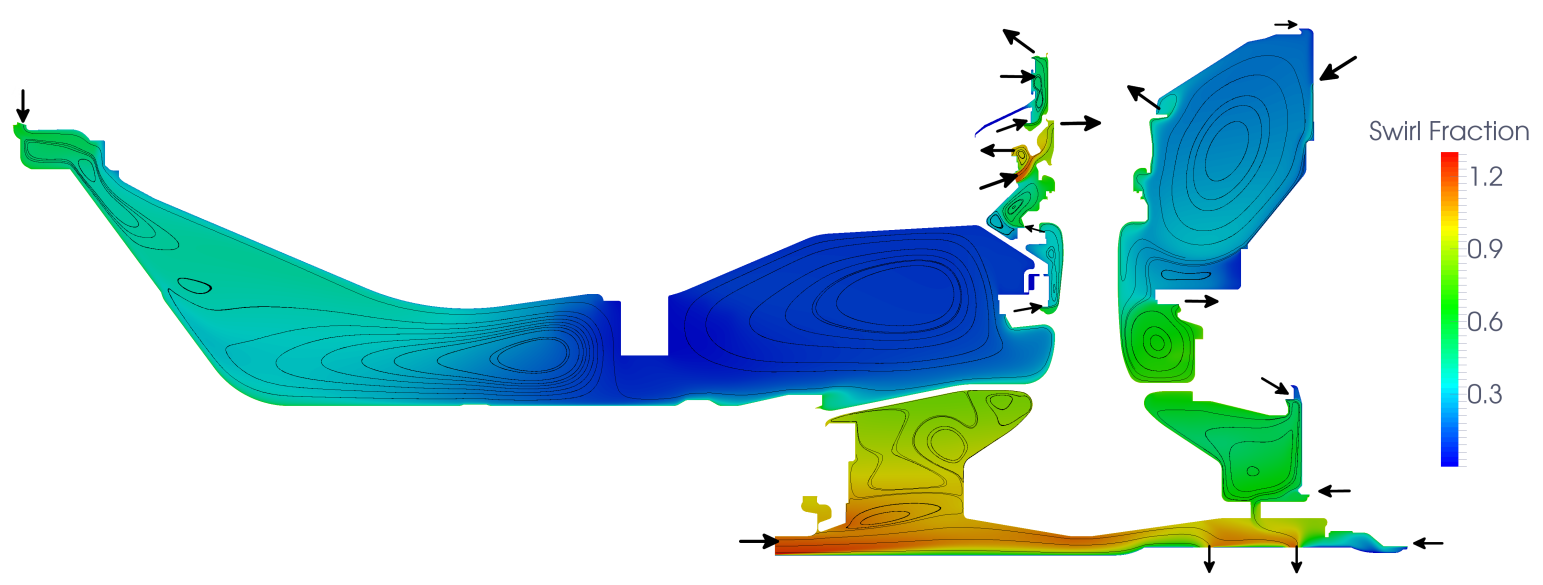

(b)

FIGURE 5. Flow field at idle condition (a) and at maximum take-off condition (b). The meridional streamlines are shown over the swirl fraction $S=U_{\theta} / \omega r$ contours. Inflow and outflow boundaries are shown with arrows.

TABLE 1. CFD models mesh resolution at two operating conditions showing the number of cells.

\begin{tabular}{ccccccc}
\hline \hline Regime & Drive Cone & PS Inner & PS Chamber & Front Rim & Rear & Bore \& Inner Rear \\
\hline Idle & 117,981 & 19,719 & 36,869 & 27,155 & 61,716 & 69,635 \\
MTO & 122,768 & 21,339 & 41,459 & 29,527 & 64,380 & 75,704 \\
\hline
\end{tabular}

as:

$$
\Delta p_{\theta}=n C_{d} \rho\left|u_{\theta}-r \omega\right|\left(u_{\theta}-r \omega\right)
$$

where $n$ is the number of protruding features per unit length.
To calibrate the aerodynamic drag force coefficients a $3 D$ model of the drive cone cavity was created with a sector size of $13.846^{\circ}$. The sector is discretized into $\sim 8 M$ cells with a $3 D$ cross-sectional grid topology replicating its $2 D$ axisymmetric counterpart. The sector geometry with the protrusions is shown in Fig. 7. Two steady stand-alone analyses were conducted on the $3 D$ model with boundary conditions representing stabilized idle and maximum take-off flow regimes and adiabatic walls. Similar computations were executed on the $2 D$ axisymmetric models 


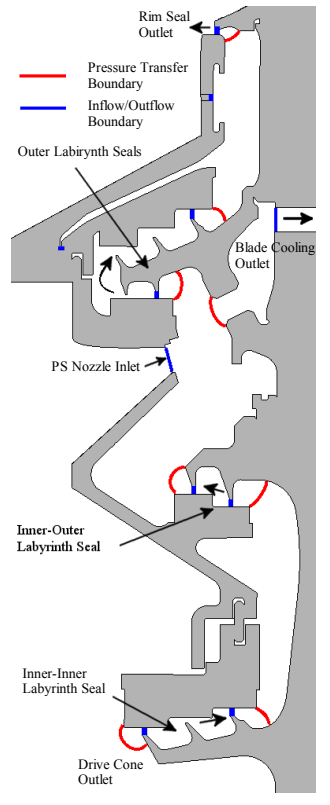

FIGURE 6. Relative locations of the inflow/outflow boundaries and the artificial boundaries created to enforce the pressure transfer conditions between $2 \mathrm{D}$ and $1 \mathrm{D}$ fluid models.

TABLE 2. Flow conditions at inlets during the idle and the MTO regimes presented in terms of the rotational Reynolds number $R e_{\theta}$, the axial Reynolds number $\operatorname{Re}_{x}$, the Mach number $M$ and the turbulent flow parameter $\lambda_{T}$.

\begin{tabular}{ccccc}
\hline \hline & $R e_{\theta}$ & $R e_{x}$ & $M$ & $\lambda_{T}$ \\
\hline Drive Cone Idle & $6.14 \times 10^{6}$ & $9.7 \times 10^{4}$ & 0.27 & 0.091 \\
Drive Cone MTO & $33.79 \times 10^{6}$ & $5.3 \times 10^{5}$ & 0.29 & 0.164 \\
\hline PS Inner Idle & $2.33 \times 10^{6}$ & $1.5 \times 10^{4}$ & 0.27 & 0.280 \\
PS Inner MTO & $12.83 \times 10^{6}$ & $0.76 \times 10^{5}$ & 0.28 & 0.500 \\
\hline PS Chamber Idle & $3.98 \times 10^{6}$ & $16.2 \times 10^{4}$ & 0.64 & 0.965 \\
PS Chamber MTO & $22.69 \times 10^{6}$ & $8.89 \times 10^{5}$ & 0.66 & 0.975 \\
\hline Front Rim Idle & $3.87 \times 10^{6}$ & $2.9 \times 10^{4}$ & 0.39 & 0.311 \\
Front Rim MTO & $22.99 \times 10^{6}$ & $1.66 \times 10^{5}$ & 0.40 & 0.252 \\
\hline Rear Idle & $3.35 \times 10^{6}$ & $0.54 \times 10^{4}$ & 0.64 & 0.094 \\
Rear MTO & $19.47 \times 10^{6}$ & $0.28 \times 10^{5}$ & 0.74 & 0.097 \\
\hline Bore Idle & $0.26 \times 10^{6}$ & $5.8 \times 10^{4}$ & 0.23 & 3.422 \\
Bore MTO & $12.00 \times 10^{6}$ & $2.4 \times 10^{5}$ & 0.28 & 4.269 \\
\hline
\end{tabular}

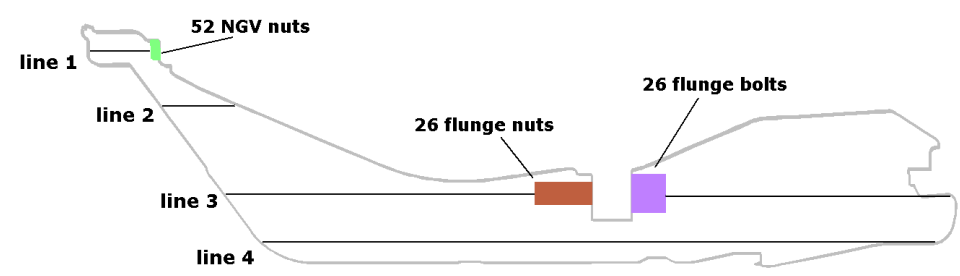

FIGURE 7. Cross section of the drive cone cavity showing the nonaxisymmetric features and the radial location of the swirl velocity probes.

with varying drag coefficients. Fig. 8 shows the swirl ratio profile comparison at four different radial locations given in Fig. 7. With the drag coefficients set to $0.75,3.0$ and 5.0 for the NGV nuts, the flange nuts and bolts respectively the simplified axisymmetric body force model appears to reproduce the swirl velocity throughout the cavity relatively well at both low and high power conditions.

\section{SIMULATION RESULTS}

To validate the multiscale heterogeneous coupling approach we carry out a transient heat transfer analysis on the described in the above test case running through a square operating cycle shown in Fig. 4. The validation process is performed by comparing the simulation predictions with transient temperature and steady pressure measurements collected during the instrumented engine test campaign. The HPT disk and surrounding regions were equipped with air and metal thermocouples and static pressure tappings. Fig. 9 shows some of the metal thermocoulpes and pressure taps locations.

Owing to significant transient changes in running seal clearances during aeroengine operation prediction of leakage and cooling flows in the cavities interconnected through the seals is a non-trivial task. Relative displacements of the seal parts can be grouped into symmetric and non-symmetric components. The former are due to the centrifugal, the pressure loads and the thermal growth differential. The latter are mainly caused by the rotor bow, bending or ovalization effects. In this simulation we do not model the non-axisymmetric effects. Instead, we employ a single geometric parameter lumping together all fins' radial clearances in the seal termed as effective clearance $C_{e f f}=\sqrt{N / \sum_{n=1}^{N} 1 / C_{n}^{2}}$, where $N$ is the number of fins in a seal. Axisymmetric relative radial displacements predicted for each fin individually are added to a pre-selected constant representing our best guess at the radial cold built clearance. The same constant is assumed for each 


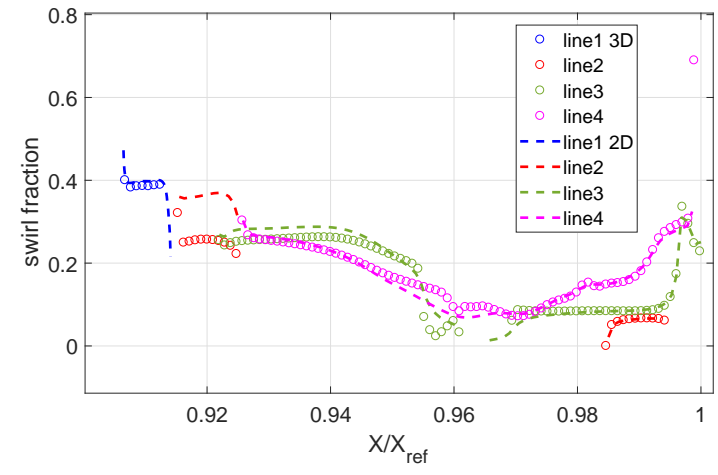

(a)

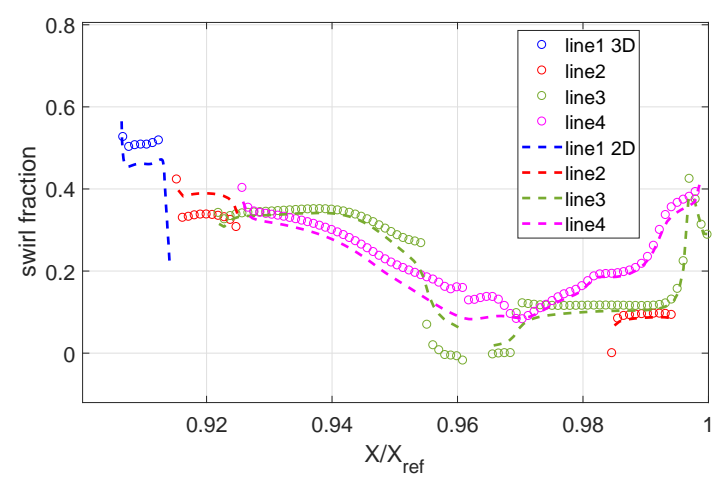

(b)

FIGURE 8. Comparison of swirl fraction predicted by $3 D$ and $2 D$ models at low power (a) and high power (b) stabilized conditions. Swirl fraction calculated at four different radii shown in Fig. 7 is plotted against the normalized axial coordinate.

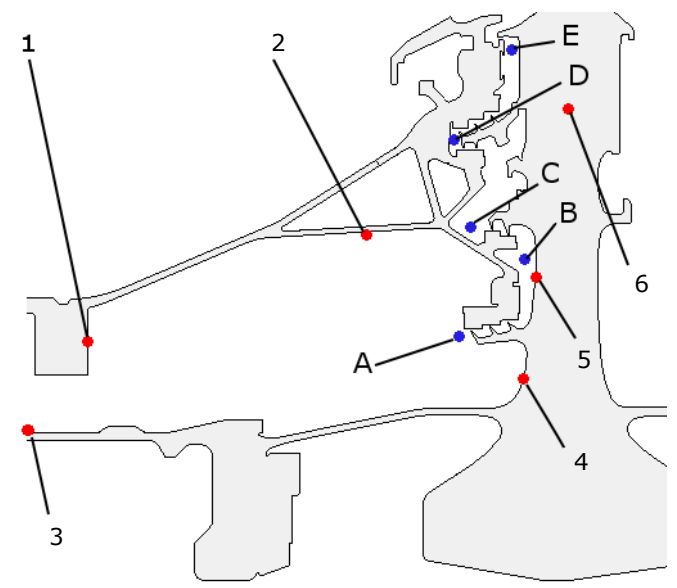

FIGURE 9. Temperature and pressure measurement positions. fin of a seal. The effective radial clearance is then converted into effective area available to gas flow in the flow network solver.

The solid FE model is numerically coupled to $1 D$ flow network model by predicting radial clearances of the 14 seals depicted in Fig. 3. Only four of them were instrumented with the pressure taps, namely the inner-inner, the inner-outer, the outerinner and the outer-outer labyrinth seals. Fig. 10 depicts the predicted radial seal clearance time histories of the four instrumented seals as a percentage of their respective cold built levels. The effects of transient clearance changes on the leakage flows

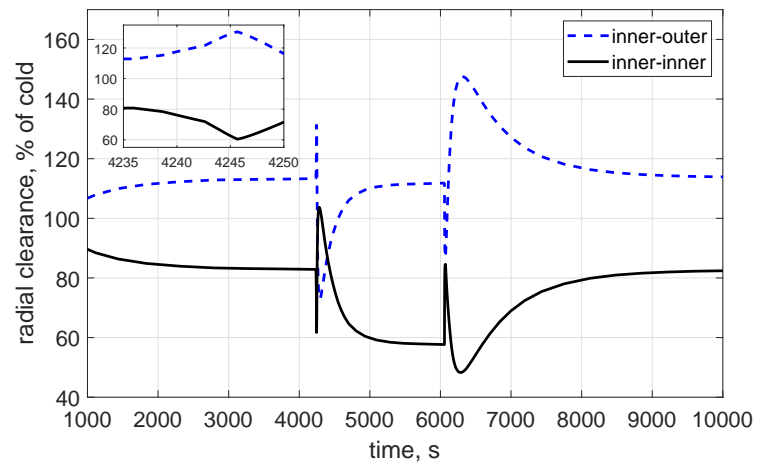

(a)

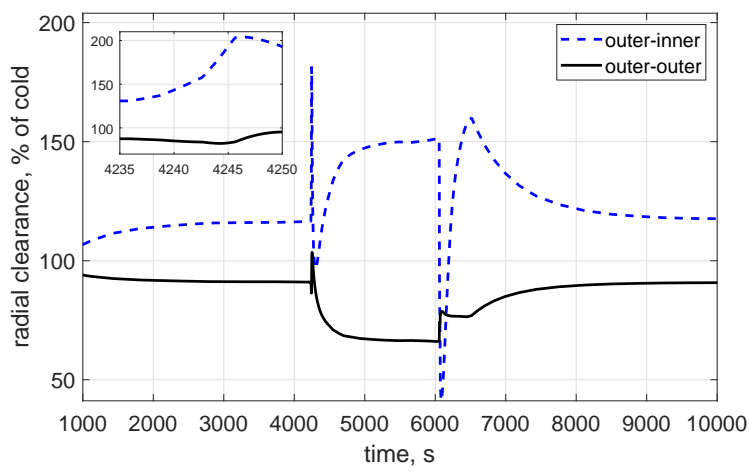

(b)

FIGURE 10. Predicted equivalent radial seal clearance of the inner seals (a) and the outer seals (b) as a percentage of the cold built clearance. The subplot shows clearances evolution zoomed to the $15 \mathrm{sec}$ acceleration ramp.

through the drive cone and the front rim seal cavities are shown in Fig. 11. Predicted mass flows appear consistent with the seal transient evolution. A sharp spike in cooling flow rates occur during the $15 s$ acceleration. We observe up to $15 \%$ higher mass flows compared to the stabilized high power level. The thermal response time is an order of magnitude longer and this due to 


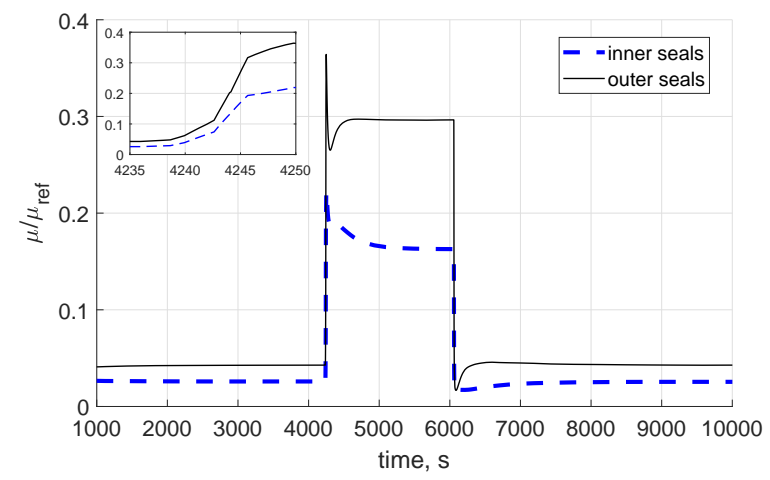

FIGURE 11. Normalized mass flow rates through the inner and the outer labyrinth seals. The subplot shows mass flow rates evolution zoomed to the $15 \mathrm{sec}$ acceleration ramp.

slower thermal growth of the massive HPT disk featureing higher thermal capacitance. It forms the rotor wall of the seals. This can be seen both in the predicted radial seal clearances and the mass flow rates.

Pressure histories calculated across the four labyrinth seals are shown in Fig. 12. In the figure the static pressure levels are normalized by the HPC delivery pressure. The transient pressure

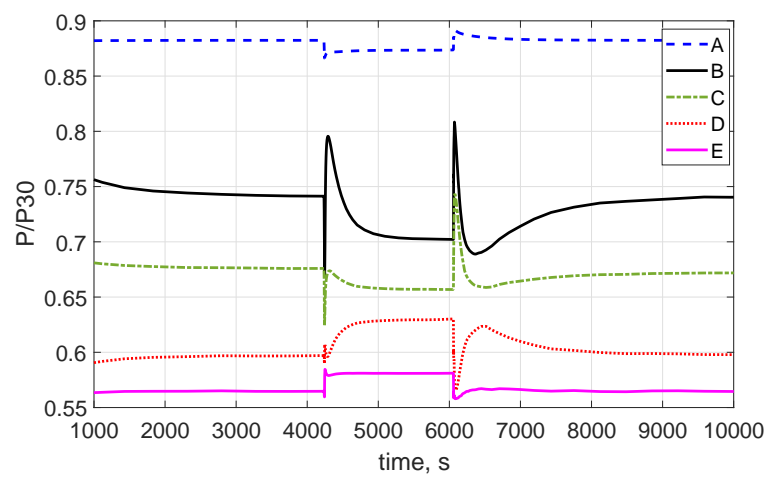

FIGURE 12. Evolution of static pressures predicted at five instrumented locations. Static pressures are non-dimensionalized by the high pressure compressor delivery pressure.

evolution reflects the structural deflections. The static pressures calculated at five instrumented tapping locations are compared against the measurement data taken at stabilized low and high power conditions in Tab. 3. The table provides detailed information on the differences between the predicted static pressures and the available measured data. The values have been made nondimensional dividing them by the corresponding levels of compressor delivery pressure. Good agreement has been achieved be- tween the experiment and simulation. Higher discrepancies have been found at idle condition around both inner seals with the maximum difference less than $1.9 \%$ in the worst case.

The heat transfer process through some of the coupled surfaces is illustrated in Figs. 13 and 14. The figures show the local convective heat transfer coefficient distributions over rotating and stationary walls in the drive cone cavity at both stabilized conditions. The distribution on the pre-swirl chamber cavity rotor wall is given in Fig. 15. The heat transfer coefficients were computed using the wall heat flux predictions $Q_{1 w}=h\left(T_{1 w}-T_{f}\right)$ and $Q_{2 w}=h\left(T_{2 w}-T_{f}\right)$ from two flow solutions obtained by imposing metal temperatures $T_{1 w}$ and $T_{2 w}=T_{1 w}+\Delta T$ correspondingly. Combining both yields a simple and robust approximation of $h t c$ $h=\left(Q_{2 w}-Q_{1 w}\right) / \Delta T$, where $\Delta T$ set to a small constant, without complicated selection of the reference bulk fluid temperature.

The magnitude of local convective heat transfer coefficient on

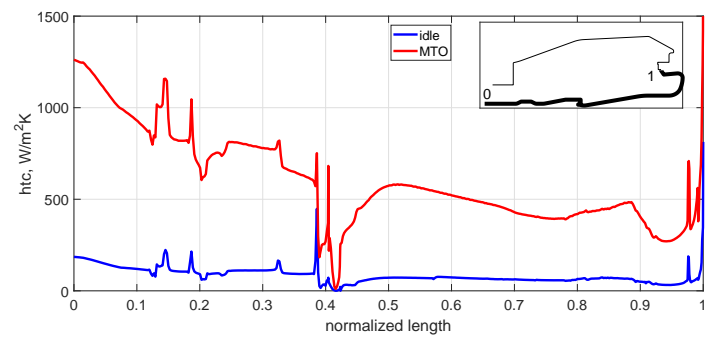

FIGURE 13. Heat transfer coefficient distribution on the rotating wall of the drive cone cavity.

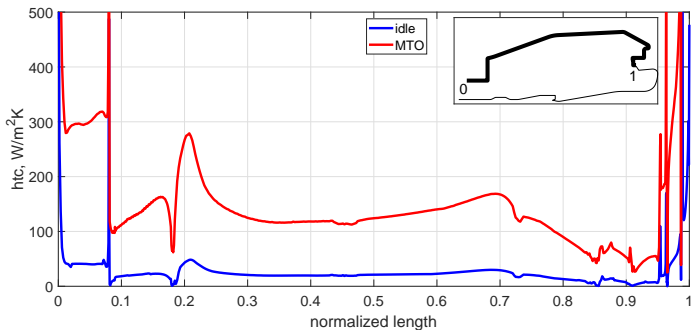

FIGURE 14. Heat transfer coefficient distribution on the stationary wall of the drive cone cavity.

the common fluid-solid interfaces is large at both engine regimes. Therefore accurate spatial and temporal predictions of boundary temperature and heat flux arising from the heat transfer processes in fluid and solid domains can only be found by simultaneous resolution for all of these processes. This justifies application of the coupled approach. 
TABLE 3. Comparison of static pressure predictions to measurement data. The values have been non-dimensionalized by the high pressure compressor delivery pressure.

\begin{tabular}{ccccccccc}
\hline Pressure tap & CFD idle & Test idle & $\Delta$ idle & $\Delta(\%)$ idle & CFD MTO & Test MTO & $\Delta$ MTO & $\Delta(\%)$ MTO \\
\hline A & 0.8727 & 0.8734 & 0.0007 & 0.08 & 0.8734 & 0.8737 & 0.0003 & 0.03 \\
B & 0.7220 & 0.7349 & 0.0029 & 0.96 & 0.7020 & 0.7102 & 0.0082 & 1.15 \\
C & 0.6762 & 0.6635 & -0.0127 & 1.90 & 0.6566 & 0.6646 & 0.0080 & 1.19 \\
D & 0.5966 & 0.5956 & -0.0010 & 0.17 & 0.6300 & 0.6322 & 0.0022 & 0.34 \\
E & 0.5646 & 0.5629 & -0.0017 & 0.30 & 0.5809 & 0.5837 & 0.0028 & 0.47 \\
\hline
\end{tabular}

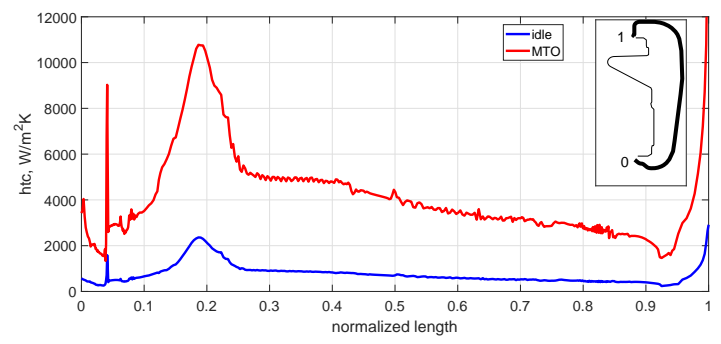

FIGURE 15. Heat transfer coefficient distribution on the rotating wall of the pre-swirl chamber cavity.

Comparison between the predicted and measured wall temperatures is shown in Fig. 16. The temperatures are normalized by the HPC delivery temperature at high power condition. The wall temperatures at both stabilized conditions are well captured for all thermocouple locations. The maximum differences with measured data are below $1 \%$ at both stabilized conditions except for the position 3 on the drive cone cavity rotor wall featuring $1.2 \%$ higher temperature at maximum power condition, which is quite close to the thermocouple measurement accuracy. During the fast acceleration and deceleration the results exhibit relatively good agreement with experimental data. Higher deviations occur after the deceleration from maximum take-off to low power condition on the turbine disk front face. Measured transient metal temperature changes show faster response than predicted by the coupled simulation by about 5\%. These discrepancies are evolving at a longer conduction time scale and can be attributed to many factors that are beyond the control and scope of the current modeling approach. One possible explanation is due to the uncertainties in running seal clearances. The total seal clearances after a maneuver is a function of the differential thermal growth and yet unaccounted for wear, erosion and axial displacements. The combined effects of these uncertainties may have caused variations in through flows creating local thermal gradients due to thermal convection. These gradients are eventually damped by diffusion.

\section{Simulation run time}

To assess the efficiency of the coupled methodology we measure the number of iterations and the CPU time required to solve the transient coupled heterogeneous problem. All simulations were conducted on a Linux cluster using 12 Dell Power Edge nodes. Each compute node hosts two $2.6 \mathrm{GHz} E 5-2670$ Intel Xeon processors, eight cores each, sharing 35 GB of RAM. The nodes are connected through Qlogic Infiniband interconnect with peak bandwidth $2.99 \mathrm{~GB} / \mathrm{s}$ and average MPI latency $1.36 \mu \mathrm{s}$. The selected square cycle transient simulation took approximately 24 days of the wall-clock time, which amounts to 3733 calls to CFD code. Iteration history of some of the averaged interface unknowns is shown in Fig. 17. For comparison, an equivalent CFD-FEA aero-thermo-mechanical simulation carried out with a frozen flow network solution on the same model takes 1528 calls to the CFD solver. Fig. 18 illustrates a typical convergence history of the mass flow, pressure, fluid and wall temperature residuals giving the number of inner iterations needed to converge at one time step of the heating ramp. For all time steps we observe similar stable and fast convergence behavior of the four coupled fields.

\section{CONCLUSION}

This work presented a novel dimensionally heterogeneous coupling technique for aero-thermo-mechanical simulation of gas turbine engine components. Defined on a restricted area of interest of the global computational domain more detailed conjugate $2 D / 3 D$ fluid and structure problems were numerically coupled to a coarser $1 D$ flow network problem of a whole engine air system. Formulation and numerical treatment of the heterogeneous interfaces allowed us to combine several geometrical multiscale models into a single nonlinear system. Recasting this system as an interface problem with subsequent application of the Anderson mixing technique stabilized and accelerated convergence resulting in a robust and efficient algorithm.

The paper illustrated excellent reliability and performance of the method applied to a realistic computationally challenging in- 


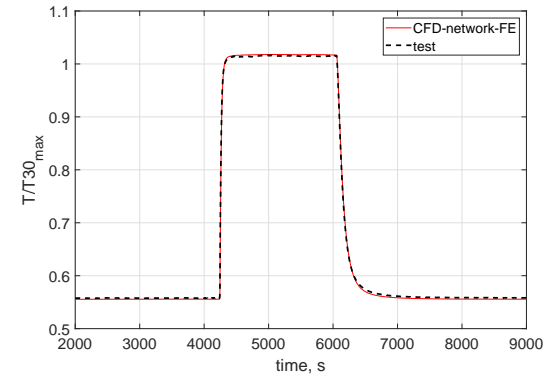

(a)

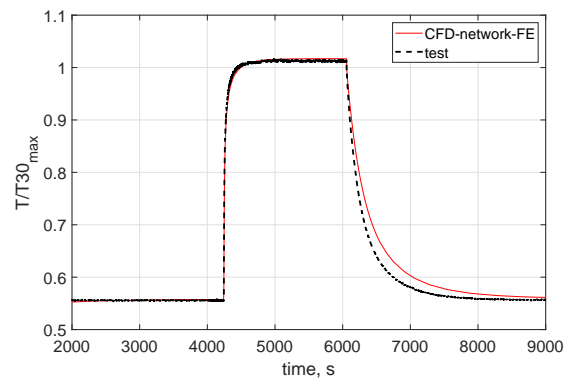

(d)

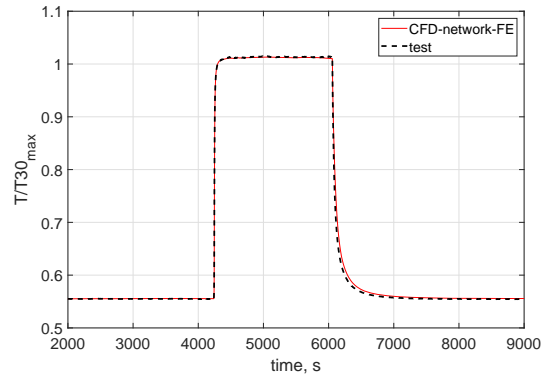

(b)

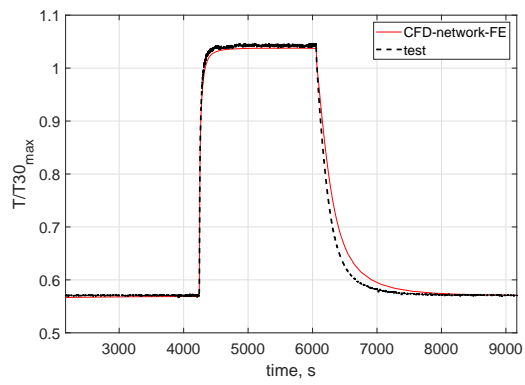

(e)

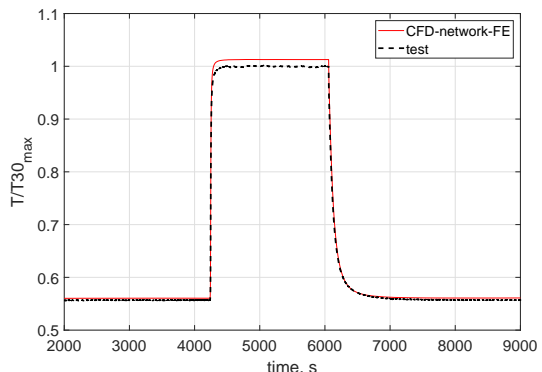

(c)

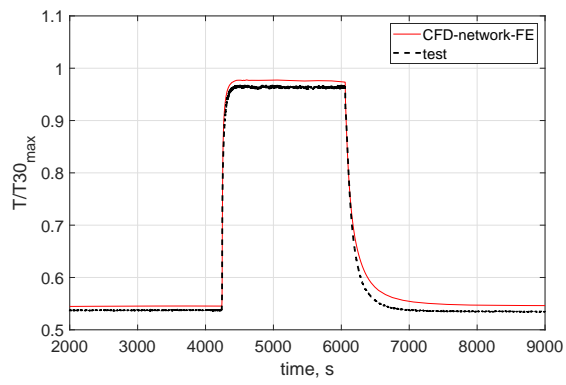

(f)

FIGURE 16. Computed metal temperatures compared to measurements for thermocouples located in the drive cone stator flange 1 (a), on the drive cone stator wall 2 (b) and on the drive cone rotor wall 3 (c) on HPT disk 4 (d), in the inner pre-swirl cavity 5 (e) and on the blade root area 6 (f).

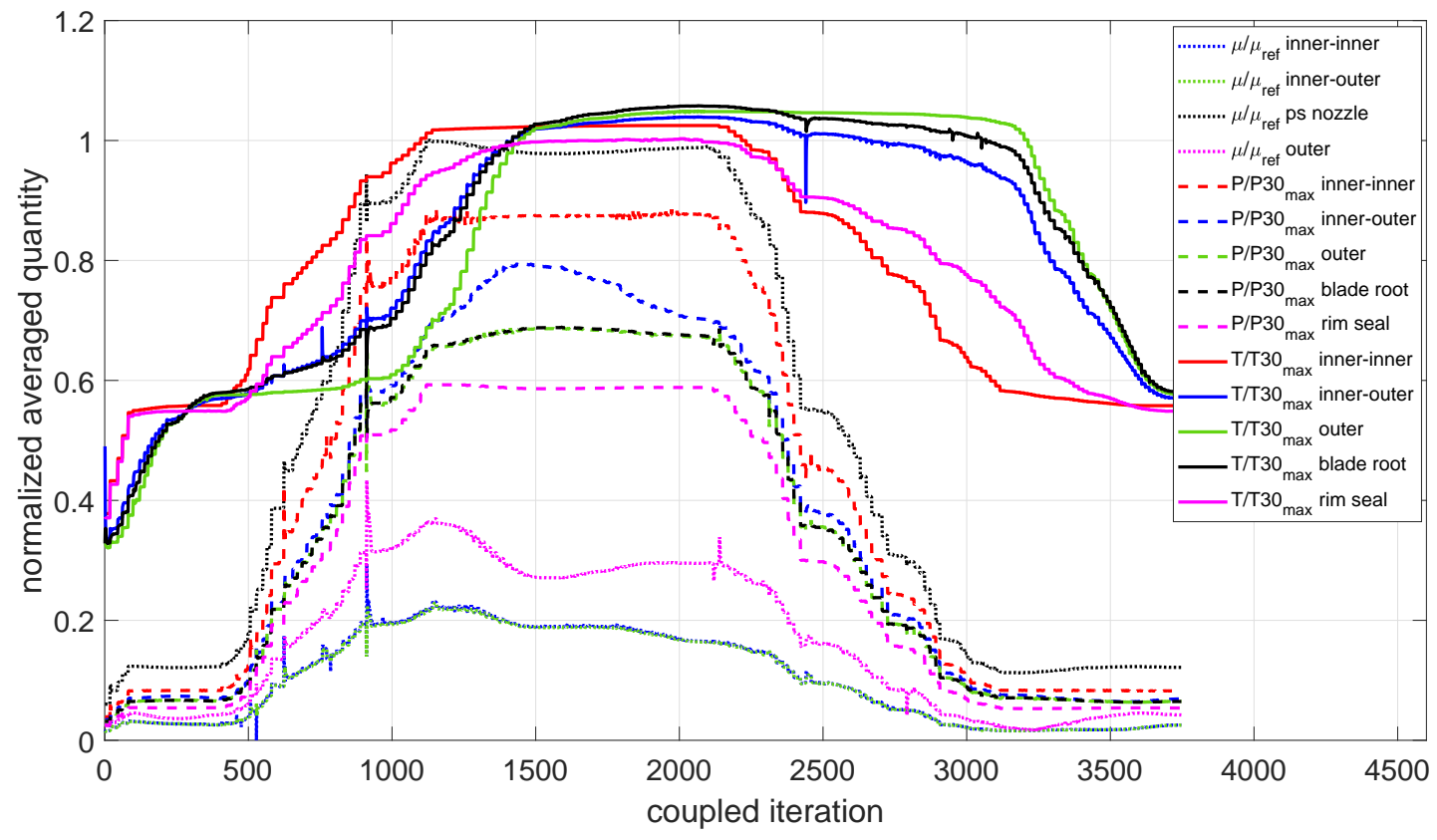

FIGURE 17. Iteration history of the flow variables averaged on the inflow-outflow boundaries depicted in Fig. 6. 


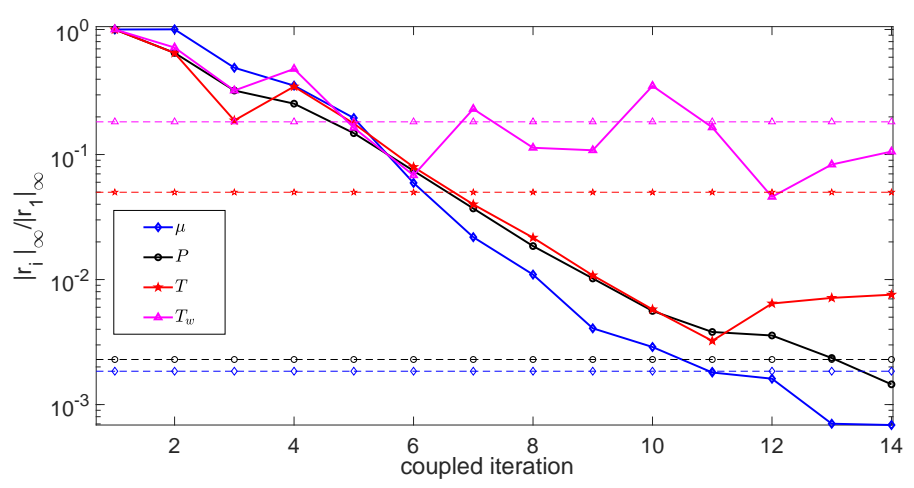

FIGURE 18. Relative residual convergence history with corresponding tolerances for a single time step.

dustrial test case. Comparisons with experimental data across available instrument locations show that the coupled simulation well captured metal temperatures and pressures throughout the entire cycle.

The proposed multiscale methodology allows to perform quantitative and qualitative studies on the interaction between local and global quantities as well as between strongly coupled aero, thermal and structural fields at significantly lower computational cost compared to fully conjugate CFD methods. This brings the accuracy that is currently beyond the capability of uncoupled stand-alone $2 D / 3 D$ and $1 D$ predictive tools. The proposed approach is an incremental step towards more realistic airsystems simulation with a great potential for preliminary design phase.

\section{ACKNOWLEDGMENT}

The authors would like to acknowledge Rolls-Royce plc and the Clean Sky Joint Technology Initiative for providing financial and technical support for this work.

\section{REFERENCES}

[1] Kutz, K., and Speer, T., 1994. "Simulation of the secondary air system of aero engines". Journal of Turbomachinery, 116(2), 04, pp. 306-315.

[2] Montenay, A., Paté, L., and Deboué, J., 2000. "Conjugate heat transfer analysis of an engine internal cavity". In ASME Turbo Expo Paper 2000-GT-0282.

[3] Verdicchio, J. A., Chew, J. W., and Hills, N. J., 2001. "Coupled fluid/solid heat transfer computation for turbine discs". In ASME Turbo Expo Paper 2001-GT-0205.

[4] Bohn, D., Heurer, T., and Kusterer, K., 2003. "Conjugate flow and heat transfer investigation of a turbo-charger - Part
I: Numerical results". In ASME Turbo Expo Paper 2003GT-38445.

[5] Verstraete, T., Alsalihi, Z., and van den Braembussche, R., 2007. "Numerical study of the heat transfer in micro gas turbines". Journal of Turbomachinery, 29, pp. 835-841.

[6] Okita, Y., and Yamawaki, S., 2002. "Conjugate heat transfer analysis of turbine rotor-stator systems". In ASME Turbo Expo Paper 2002-GT-30615.

[7] Sun, Z., Chew, J. W., Hills, N. J., Lewis, L., and Mabilat, C., 2011. "Coupled aerothermomechanical simulation for a turbine disk through a full transient cycle". Journal of Turbomachinery, 134(1), May, pp. 011014-011014-11.

[8] Ganine, V., Javiya, U., Hills, N., and Chew, J., 2012. "Coupled fluid-structure transient thermal analysis of a gas turbine internal air system with multiple cavities". Journal of Engineering for Gas Turbines and Power, 134(10), Aug., pp. 102508-102508-8.

[9] Altuna, A., Chaquet, J., Corral, R., Gisbert, F., and Pastor, G., 2013. "Application of a fast loosely coupled fluid/solid heat transfer method to the transient analysis of low-pressure-turbine disk cavities". In ASME Turbo Expo Paper 2013-GT-95426.

[10] Errera, M.-P., Lazareff, M., Garaud, J.-D., Soubrie, T., Douta, C., and Federici, T., 2017. "A coupling approach to modeling heat transfer during a full transient flight cycle". International Journal of Heat and Mass Transfer, 110, pp. $587-605$.

[11] Maffulli, R., He, L., Stein, P., and Marinescu, G., 2018. "Fast conjugate heat transfer simulation of long transient flexible operations using adaptive time stepping". Journal of Turbomachinery, 140(9), 08. 091005.

[12] Amirante, D., Hills, N., and Barnes, C., 2012. "Thermomechanical finite element anaysis/computational fluid dynamics coupling of an interstage seal cavity using torsional spring analogy". Journal of Turbomachinery, 134(5), pp. 051015-1-051015-9.

[13] Ganine, V., Amirante, D., and Hills, N. J., 2016. “Aerothermo-mechanical modelling and validation of transient effects in a high pressure turbine internal air system". In ASME Turbo Expo Paper 2016-GT-57739.

[14] Muller, Y., $2009 . \quad$ "Integrated fluid networkthermomechanical approach for the coupled analysis of a jet engine". In ASME Turbo Expo Paper 2009-GT-59104.

[15] Giuntini, S., Andreini, A., and Facchini, B., 2019. "Finite element transient modeling for aero-thermo-mechanical analysis of whole gas turbine engine". In ASME Turbo Expo Paper 2019-GT-91278.

[16] Ganine, V., Hills, N., Miller, M., Barnes, C., Curzons, S., Turner, L., and Smout, P., 2015. "Implicit heterogeneous 1D/2D coupling for aero-thermo-mechanical simulation of secondary air systems". In ASME Turbo Expo Paper 2015GT-43406. 
[17] Mohamed, S. N., Chew, J. W., and Hills, N. J., 2015. "Simplified protrusion drag and heat transfer modelling of bolts on a rotating disc". In ASME Turbo Expo Paper 2015-GT43501.

[18] Quarteroni, A., Veneziani, A., and Vergara, C., 2016. "Geometric multiscale modeling of the cardiovascular system, between theory and practice". Computer Methods in Applied Mechanics and Engineering, 302, Apr., pp. 193-252.

[19] Formaggia, L., Gerbeau, J., Nobile, F., and Quarteroni, A., 2002. "Numerical Treatment of Defective Boundary Conditions for the Navier-Stokes Equations". SIAM Journal on Numerical Analysis, 40(1), Jan., pp. 376-401.

[20] Errera, M.-P., and Baqué, B., 2013. "A quasi-dynamic procedure for coupled thermal simulations". International Journal for Numerical Methods in Fluids, 72(11), Aug., pp. 1183-1206.

[21] Giles, M., 1997. "Stability analysis of numerical interface conditions in fluid-structure thermal analysis". International Journal for Numerical Methods in Fluids, 25 , pp. 421-436.

[22] Roe, B., Haselbacher, A., and Geubelle, P., 2007. "Stability of fluid-structure thermal simulations on moving grids". International Journal for Numerical Methods in Fluids, $\mathbf{5 4}$ pp. 1097-1117.

[23] Knoll, D., and Keyes, D., 2004. "Jacobian-free NewtonKrylov methods: A survey of approaches and applications". Journal of Computational Physics, 193, pp. 357-397.

[24] Anderson, D., 1965. "Iterative procedures for nonlinear integral equations". Journal of the Association of Computing Machinery, 12, pp. 547-560.

[25] Ganine, V., Hills, N. J., and Lapworth, B. L., 2013. "Nonlinear acceleration of coupled fluid-structure transient thermal problems by Anderson mixing". International Journal for Numerical Methods in Fluids, 71(8), Mar., pp. 939-959.

[26] Armstrong, I., and Edmunds, T., 1989. "Fully automatic analysis in the industrial environment". International Conference on Quality Assurance and Standards, NAFEMS.

[27] Dixon, J., Verdicchio, J., Benito, D., Karl, A., and Tham, K., 2004. "Recent developments in gas turbine component temperature prediction methods, using computational fluid dynamics and optimization tools, in conjunction with more conventional finite element analysis techniques". In Proceedings of the Institution of Mechanical Engineers, Journal of Power and Energy, Vol. 128(4).

[28] Javiya, U., Chew, J. W., Hills, N. J., Zhou, L., Wilson, M., and Lock, G. D., 2012. "CFD analysis of flow and heat transfer in a direct transfer preswirl system". Journal of Turbomachinery, 134(3), p. 031017.

[29] Javiya, U., Chew, J., Hills, N., Dullenkopf, K., and Scanlon, T., 2013. "Evaluation of computational fluid dynamics and coupled fluid-solid modeling for a direct transfer preswirl system". Journal of Engineering for Gas Turbines and Power, 135(5), p. 051501.

[30] Moinier, P., 1999. "Algorithm developments for an unstructured viscous flow solver". PhD thesis, Oxford University. 